\title{
Invariant Measures of Gaussian Type for 2D Turbulence
}

\section{Hakima Bessaih \& Benedetta Ferrario}

Journal of Statistical Physics

1

ISSN 0022-4715

Volume 149

Number 2

J Stat Phys (2012) 149:259-283

DOI 10.1007/s10955-012-0601-z
Volume 149 - Number 2 - October 2012

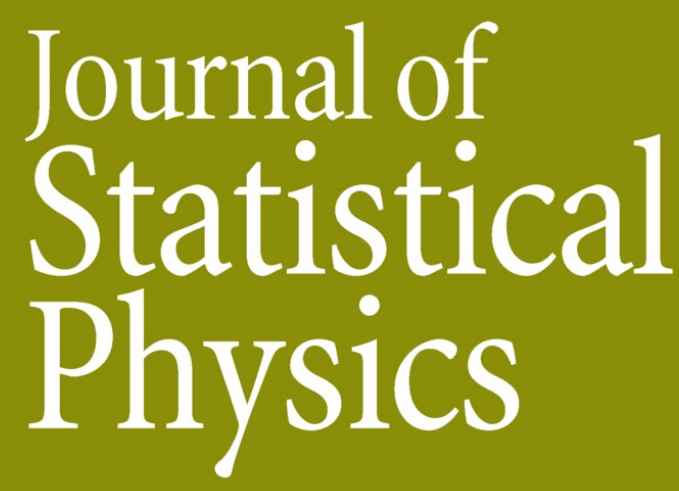

Journal of Statistical Physics

Available $+\frac{7}{4}$

online

$10955 \cdot$ ISSN 0022-4715 149(2) 203-384 (2012)

空 Springer 
Your article is protected by copyright and all rights are held exclusively by Springer Science +Business Media New York. This e-offprint is for personal use only and shall not be selfarchived in electronic repositories. If you wish to self-archive your work, please use the accepted author's version for posting to your own website or your institution's repository. You may further deposit the accepted author's version on a funder's repository at a funder's request, provided it is not made publicly available until 12 months after publication. 


\title{
Invariant Measures of Gaussian Type for 2D Turbulence
}

\author{
Hakima Bessaih • Benedetta Ferrario
}

Received: 16 April 2012 / Accepted: 15 September 2012 / Published online: 26 September 2012

(C) Springer Science+Business Media New York 2012

\begin{abstract}
Gaussian measures $\mu^{\beta, \nu}$ are associated to some stochastic 2D models of turbulence. They are Gibbs measures constructed by means of an invariant quantity of the system depending on some parameter $\beta$ (related to the $2 \mathrm{D}$ nature of the fluid) and the viscosity $v$. We prove the existence and the uniqueness of the global flow for the stochastic viscous system; moreover the measure $\mu^{\beta, v}$ is invariant for this flow and is the unique invariant measure. Finally, we prove that the deterministic inviscid equation has a $\mu^{\beta, \nu}$-stationary solution (for any $v>0)$.
\end{abstract}

Keywords Gibbs measures · Stochastic analysis · Invariant measures · 2D turbulence

\section{Introduction}

The goal of this paper is to study a class of mathematical models related to 2D fluids through their statistical properties and in particular their invariant measures. From a theoretical point of view, 2D turbulence is not simply a reduced dimensional version of 3D turbulence because a completely different phenomenology arises from new conservation laws in two dimensions, energy and enstrophy. Energy and enstrophy input are usually implemented numerically using a Gaussian stochastic forcing which is white noise in time. We refer to the paper [6], where an overview of 2D turbulence is described thoroughly.

There is an extensive literature about the existence and uniqueness of solutions of the stochastic 2D Navier-Stokes equation. Its long time behavior has also been extensively studied, including the existence and uniqueness of invariant measures (see, e.g.,

H. Bessaih

Department of Mathematics, University of Wyoming, Dept. 3036, 1000 East University Avenue,

Laramie, WY 82071, USA

e-mail: bessaih@uwyo.edu

B. Ferrario (凶)

Dipartimento di Matematica, Università di Pavia, via Ferrata 1, 27100 Pavia, Italy

e-mail: benedetta.ferrario@unipv.it 
[20] and the references therein including the results of Flandoli, Hairer, Kuksin, Kupiainen, Maslowski, Mattingly, Shirikyan, Sinai).

As far as the 2D Euler equation is concerned, a recent paper [7] constructs invariant measures of general types including the Gaussian measures we are interested in. However, the techniques used in [7] are different from ours and the measures are described through a variational formulation.

The study of qualitative properties of the invariant measures is an interesting problem both from the mathematical and physical point of view, but many open questions still wait for an answer. Among the non trivial invariant measures there is a Gaussian measure, called the Gibbs measure of the enstrophy (see $[1,2,13,16]$ ), which is a stationary measure both for the 2D Navier-Stokes with additive space-time white noise and for the 2D deterministic Euler equation. However, it is not known if this is the unique invariant measure.

Our contribution in this paper is to be able to prove existence and uniqueness of an invariant measure of Gaussian type for a very general model that does not include the 2D Navier-Stokes equation but that has all the peculiar features of 2D fluids, like conservation of energy and enstrophy. We point out that the Gaussian invariant measure that we consider here is not the Gibbs measure of the enstrophy considered for the 2D stochastic NavierStokes or deterministic 2D Euler equation in previous papers [1, 2, 4, 13, 16], but has a more regular support. In particular, the support of this measure is a Sobolev space of non negative exponent.

From the mathematical point of view, the equations of fluid dynamics or of models related to turbulence can be represented as an abstract stochastic evolution equation in a Hilbert space of the following form

$$
d u(t)+[v A u(t)+B(u(t), u(t))] d t=\sqrt{Q} d w(t),
$$

where $w$ is a cylindrical Wiener process and $Q$ is a linear operator; the coefficient $v \geq 0$ is the viscosity. More details on the functional setting will be given in the next section.

For the abstract model (1) under suitable assumptions on the operators $A, B$ and $Q$, we prove that it is well posed in the support of the Gaussian measure $\mu^{\beta, \nu}$; this is done following the technique of [20], differently from $[2,13,16]$. Then, we prove that $\mu^{\beta, v}$ is an invariant measure, and that there exists at most one invariant measure; uniqueness of the invariant measure is proved by means of Girsanov theorem. Finally, for the deterministic and inviscid model $(Q=0, v=0)$, we prove that there exists a stationary solution, whose marginal at fixed time is this Gaussian measure.

We provide an example of equation of the form (1) for which our results hold: the shell model of turbulence given in Sect. 4 with the peculiar properties of 2D fluids. Shell models of turbulence have been introduced as a simplified phenomenological model of fluid dynamic equations in order to investigate a number of properties which are out of reach at present for the more realistic models. This is because, although departing from reality, they capture some essential statistical properties and features of turbulent flows. On the other side, even if the 2D Navier-Stokes/Euler equations can be formulated at the abstract level as Eq. (1), they do not satisfy all the assumptions of our theorems (see Remark 4.2 in Sect. 4). Our hope is to weaken these assumptions and still be able to use the analysis used here.

As far as the content of this paper is concerned, in Sect. 2 we introduce the operators associated to the model (1) with their properties and the Gibbs measures $\mu^{\beta, \nu}$. We introduce the (linear) Ornstein-Uhlenbeck equation with a suitable noise so that $\mu^{\beta, v}$ is its unique invariant measure. In Sect. 3, we deal with the viscous stochastic case; we prove the existence 
and uniqueness of strong solutions and that $\mu^{\beta, v}$ is the unique invariant measure. Moreover, some ergodic properties of this measure with its rate of convergence are shown. In Sect. 4, we introduce a particular example, shell models of turbulence with an emphasis on the Sabra model. The coefficient $\beta$ characterizing the measure $\mu^{\beta, v}$ will be related to the coefficients $a$ and $b$ of the Sabra model through condition (59). Section 5 is devoted to the deterministic inviscid model, in particular we present our results for the inviscid Sabra model with $\beta=1$. For any $v>0$ we prove the existence of a stationary process whose law at any fixed time is $\mu^{1, \nu}$.

\section{Introduction to the Model and Functional Setting}

\subsection{Operators and Spaces}

Let $(H,|\cdot|)$ be a real separable Hilbert space endowed with an inner product denoted by $(\cdot, \cdot)$, and $A$ an unbounded self-adjoint positive linear operator on $H$ with compact resolvent. We denote by $0<\lambda_{1} \leq \lambda_{2} \leq \cdots$ the eigenvalues of $A$ and by $e_{1}, e_{2}, \ldots$ a complete orthonormal system in $H$ given by the eigenfunctions of the operator $A$

$$
A e_{n}=\lambda_{n} e_{n} .
$$

We have that $\lim _{n \rightarrow \infty} \lambda_{n}=\infty$.

Let $H_{n}=\operatorname{span}\left\{e_{1}, e_{2}, \ldots, e_{n}\right\}$ and $\Pi_{n}$ the projector operator onto $H_{n}$.

For any $\alpha \in \mathbb{R}$ we can define the power operators $A^{\alpha}$ as

$$
A^{\alpha} x=\sum_{n=1}^{\infty} \lambda_{n}^{\alpha}\left(x, e_{n}\right) e_{n}, \quad D\left(A^{\alpha}\right)=\left\{x=\sum_{n=1}^{\infty} x_{n} e_{n}: \sum_{n=1}^{\infty} \lambda_{n}^{2 \alpha} x_{n}^{2}<\infty\right\} .
$$

We set

$$
H^{\alpha}=D\left(A^{\alpha / 2}\right) .
$$

Each $H^{\alpha}$ is a Hilbert space with scalar product $\langle u, v\rangle_{H^{\alpha}}:=\left(A^{\alpha / 2} u, A^{\alpha / 2} v\right)$. We denote by $\|\cdot\|_{\alpha}$ the norm in $H^{\alpha}$ and by $\langle\cdot, \cdot\rangle$ the $H^{\alpha}-H^{-\alpha}$ duality paring.

Let $B: H \times H \rightarrow H^{-1}$ be a bilinear operator; we assume that there exists a positive constant $c$ such that

$$
\|B(u, v)\|_{-1} \leq c|u \| v| .
$$

We consider the finite dimensional approximation of the bilinear operator $B$; this is the bilinear operator $B^{M}$ defined as

$$
B^{M}(u, v)=\Pi_{M} B\left(\Pi_{M} u, \Pi_{M} v\right)
$$

for any $M \in \mathbb{N}$. For each $B^{M}$ we have the same estimate as (2) (with the constant $c$ independent of $M$ ).

For any $v>0$, let $\mu^{\beta, v}$ be the Gaussian measure $\mathcal{N}\left(0, \frac{1}{v} A^{-\beta}\right)$ (see, e.g., [14, 23]). Heuristically

$$
\mu^{\beta, v}(d u)=“ \frac{1}{Z} e^{-v\|u\|_{\beta}^{2}} d u ”
$$

where $Z$ is a normalization constant to make $\mu^{\beta, v}$ a probability measure. 
Besides the basic properties of the operators $A$ and $B$ given above, we present other important assumptions.

Condition (C1): For any $v>0$, the operator $v A$ generates an analytic semigroup of contractions in $H$, i.e. for any $p>0$ there exists $c_{p, v}>0$ such that

$$
\left|A^{p} e^{-v A t} x\right| \leq \frac{c_{p, v}}{t^{p}}|x| \quad \forall t>0, x \in H .
$$

Condition (C2): The bilinear operator $B$ satisfies the following properties:

(i) $\langle B(u, v), w\rangle=-\langle B(u, w), v\rangle$

(ii) $\exists \beta>0$ such that $\left\langle B(u, u), A^{\beta} u\right\rangle=0$

for any $u, v, w$ giving meaning to the above relationships.

Condition (C3): For each $n$, set $B_{n}(u, v)=\left\langle B(u, v), e_{n}\right\rangle$. Then, for some $\beta>0$ we have

$$
\int\left|B_{n}(x, x)\right|^{2} \mu^{\beta, v}(d x)<\infty \quad \forall n
$$

and $B_{n}(x, x)$ independent of $x_{n}$ (where $\left.x=\sum_{n} x_{n} e_{n}\right)$. Moreover, for some $\beta \in(0,1]$

$$
\lim _{M \rightarrow \infty} \sum_{n=1}^{M} \int\left|\left\langle B^{M}(x, x)-B(x, x), e_{n}\right)\right|^{2} \mu^{\beta, v}(d x)=0 .
$$

Condition (C4): There exist $\beta>0$ and $\alpha \in[0, \beta)$ such that the embedding $H^{\beta} \subset H^{\alpha}$ is Hilbert-Schmidt, i.e.

$$
\sum_{n=1}^{\infty} \lambda_{n}^{\alpha-\beta}<\infty
$$

Condition (C5): for $\alpha$ and $\beta$ given in (C2) and (C4), $B: H^{\alpha} \times H^{\alpha} \rightarrow H^{\beta-1}$ is a continuous operator, i.e.

$$
\|B(u, v)\|_{\beta-1} \leq c\|u\|_{\alpha}\|v\|_{\alpha} \quad \forall u, v \in H^{\alpha} .
$$

Moreover, if $\alpha>0$ we assume

$$
\|B(u, v)\|_{\alpha-1} \leq c|u|\|v\|_{\alpha} \quad \forall u \in H, v \in H^{\alpha} .
$$

Remark 2.1 (i) We have the relationships corresponding to assumption (C2):

$$
\begin{gathered}
\left(B^{M}(u, v), w\right)=-\left(B^{M}(u, w), v\right), \\
\left(B^{M}(u, u), A^{\beta} u\right)=0 .
\end{gathered}
$$

(ii) From (C2 i) we have

$$
\langle B(u, v), v\rangle=0 \quad \text { and } \quad\left(B^{M}(u, v), v\right)=0 .
$$

(iii) By means of the bilinearity and of estimate (6) we have

$$
\lim _{M \rightarrow \infty}\left\|B^{M}(u, v)-B(u, v)\right\|_{\beta-1}=0 \quad \forall u, v \in H^{\alpha} .
$$


(iv) Since $\alpha \geq 0$, the inequality (7) implies

$$
\|B(u, v)\|_{\alpha-1} \leq c\|u\|_{\alpha}\|v\|_{\alpha} \quad \forall u, v \in H^{\alpha} .
$$

Moreover,

$$
\lim _{M \rightarrow \infty}\left\|B^{M}(u, v)-B(u, v)\right\|_{\alpha-1}=0 \quad \forall u, v \in H^{\alpha} .
$$

(v) Assumption (C4) implies that the space $H^{\alpha}$ has full measure $\mu^{\beta, v}$, i.e. $\mu^{\beta, v}\left(H^{\alpha}\right)=1$. However, for Gaussian measures in infinite dimensional spaces we have $\mu^{\beta, v}\left(H^{\beta}\right)=0$ (see, e.g., [23]). Also

$$
\int|u|^{2} d \mu^{\beta, \nu}<\infty \quad \int\|u\|_{\beta}^{2} d \mu^{\beta, \nu}=+\infty .
$$

(vi) Assumptions (C4) and (C5) imply that (4) holds.

We denote by $\mathcal{L}^{p}\left(\mu^{\beta, \nu}\right)$ the space of measurable functions $\phi$ defined in the support of the measure $\mu^{\beta, v}$ and such that $\int|\phi|^{p} d \mu^{\beta, v}<\infty$.

\subsection{The Equations}

Set $Q=2 A^{1-\beta}$ in (1), that is we consider the following nonlinear stochastic equation

$$
d u(t)+[v A u(t)+B(u(t), u(t))] d t=\sqrt{2 A^{1-\beta}} d w(t) .
$$

In addition we deal with the inviscid and deterministic equation

$$
\frac{d u}{d t}(t)+B(u(t), u(t))=0
$$

and with the viscous linear stochastic equation

$$
d z(t)+v A z(t) d t=\sqrt{2 A^{1-\beta}} d w(t)
$$

(10) implies a formal law of conservation of $E(t)=\frac{1}{2}|u(t)|^{2}$ in Eq. (14). We recall that in hydrodynamics the quantity $E(t)$ is the energy, which is a conserved quantity in the motion of incompressible inviscid fluids.

Relationship (ii) in assumption (C2) implies that $S_{\beta}(t)=\frac{1}{2}\|u(t)\|_{\beta}^{2}$ is a conserved quantity for Eq. (14), that is formally we have

$$
\frac{d S_{\beta}}{d t}(t)=\left\langle\dot{u}(t), A^{\beta} u(t)\right\rangle=-\left\langle B(u(t), u(t)), A^{\beta} u(t)\right\rangle=0 .
$$

The Gaussian measure $\mu^{\beta, \nu}=\mathcal{N}\left(0, \frac{1}{v} A^{-\beta}\right)$ can be described heuristically as

$$
\mu^{\beta, v}(d u)=“ \frac{1}{Z} e^{-v S_{\beta}(u)} d u ” .
$$

Therefore it makes sense to see if the measure $\mu^{\beta, v}$, described by means of the invariant quantity $S_{\beta}$, is a stationary statistical solution for the inviscid equation (14). To this end, we will first prove that $\mu^{\beta, v}$ is a stationary measure for the stochastic viscous equation (13) looking for a dynamics in the space $H^{\alpha}$ of full measure $\mu^{\beta, v}$. However, the basic stochastic 
case to deal with is the linear equation (15) for which we recall well known properties (see [14]). Let us mention here that the stochastic forcing term is infinite dimensional and acts on all the components $e_{n}$ of $H$.

Proposition 2.2 Let assumptions (C1) and (C4) be satisfied.

Then, for any $z(0) \in H^{\alpha}$ there exists a unique strong solution to Eq. (15) such that

$$
z \in C\left([0, T] ; H^{\alpha}\right) \quad \mathbb{P} \text {-a.s. }
$$

The stationary process solving Eq. (15) is

$$
\zeta(t)=\sqrt{2} \int_{-\infty}^{t} e^{-v(t-s) A} \sqrt{A^{1-\beta}} d w(s)
$$

and the law of $\zeta(t)$ is $\mu^{\beta, v}$ for any time $t$.

Remark 2.3 (i) For 2D Euler equation with $\beta=1$, the energy $E$ is finite $\mu^{1, v}$-a.s., whereas the enstrophy $S_{1}$ is infinite $\mu^{1, v}$-a.s.

(ii) It is possible to define a formally invariant measure constructed by means of the invariant $E=\frac{1}{2}|u|^{2}$

$$
\mu^{\beta, \nu}(d u)=“ \frac{1}{Z} e^{-v E(u)} d u ” .
$$

This has been already considered for shell models of turbulence in [4].

\section{Stochastic Viscous Models}

We consider Eq. (13); first we prove that there exists a unique solution for any initial data in $H^{\alpha}$. The solution is strong in the probabilistic sense and uniqueness is in pathwise sense. Moreover, we show that $\mu^{\beta, v}$ is the unique invariant measure associated with this stochastic equation.

\subsection{Strong Solution}

We look for dynamics in the state space $H^{\alpha}$ with $0 \leq \alpha<\beta$ fulfilling assumptions (C1), (C2), (C4), (C5). We consider any finite time interval $[0, T]$.

Theorem 3.1 Let assumptions (C1), (C2), (C4) and (C5) be satisfied.

Then, for any $u(0) \in H^{\alpha}$, there exists a unique solution $u$ to Eq. (13) such that

$$
u \in C\left([0, T] ; H^{\alpha}\right) \quad \mathbb{P} \text {-a.s. }
$$

Moreover, the process $u$ is a Markov process, Feller in $H^{\alpha}$.

We divide the proof in three steps in the following subsections. 


\subsubsection{Existence of Strong Solutions}

We use a well known trick to study a stochastic semilinear equation with additive noise (see [20]).

We set $v=u-z$. Then

$$
\frac{d v}{d t}(t)+v A v(t)+B(v(t)+z(t), v(t)+z(t))=0
$$

with $v(0)=u(0)-z(0)$. Set $z(0)=0$.

Proposition 3.2 We consider the same assumptions as in Theorem 3.1. Let $v(0) \in H^{\alpha}$. Then there exists a solution to Eq. (16) such that

$$
v \in C\left([0, T] ; H^{\alpha}\right) \cap L^{2}\left(0, T ; H^{1+\alpha}\right) \quad \mathbb{P} \text {-a.s. }
$$

Proof We proceed pathwise. We want to prove that Eq. (16) has a solution $\mathbb{P}$-a.s. In a standard way (see [27]), we consider first the finite dimensional Galerkin problem associated to (16), that is

$$
\frac{d v^{M}}{d t}(t)+v A v^{M}(t)+B^{M}\left(v^{M}(t)+z^{M}(t), v^{M}(t)+z^{M}(t)\right)=0
$$

with $v^{M}(0)=\Pi_{M} v(0)$ and $z^{M}(t)=\Pi_{M} z(t)$. It is a classical result (see [27]) that (17) has a global solution $v^{M} \in C\left([0, T] ; H_{M}\right)$. We are going to get a priori estimates on the sequence $\left\{v^{M}\right\}_{M}$, which are independent of $M$. The estimates will allow to extract a subsequence converging in a suitable way.

We take the scalar product of the left hand side of Eq. (17) with $v^{M}$ in $H$; we get

$$
\begin{aligned}
\frac{1}{2} \frac{d}{d t}\left|v^{M}\right|^{2}+v\left\|v^{M}\right\|_{1}^{2} & =-\left(B^{M}\left(v^{M}+z^{M}, v^{M}+z^{M}\right), v^{M}\right) \\
& =-\left(B^{M}\left(v^{M}+z^{M}, z^{M}\right), v^{M}\right) \quad \text { by (10) } \\
& \leq\left\|B\left(v^{M}+z^{M}, z^{M}\right)\right\|\left\|_{-1}\right\| v^{M} \|_{1} \\
& \leq c\left|v^{M}+z^{M}\left\|z^{M} \mid\right\| v^{M} \|_{1} \quad\right. \text { by (2) } \\
& \leq \frac{v}{2}\left\|v^{M}\right\|_{1}^{2}+\frac{c_{v}}{2}\left|z^{M}\right|^{2}\left|v^{M}\right|^{2}+\frac{c_{v}}{2}\left|z^{M}\right|^{4}
\end{aligned}
$$

by Young inequality, for some positive constant $c_{\nu}$. Henceforth, we denote by $c_{\nu}$ a generic constant depending on $v$, but independent of $M$.

We use $\left|\Pi_{M} x\right| \leq|x|$. Therefore

$$
\frac{d}{d t}\left|v^{M}\right|^{2}+v\left\|v^{M}\right\|_{1}^{2} \leq c_{v}|z|^{2}\left|v^{M}\right|^{2}+c_{\nu}|z|^{4}
$$

Hence, Gronwall inequality applied to

$$
\frac{d}{d t}\left|v^{M}\right|^{2} \leq c_{\nu}|z|^{2}\left|v^{M}\right|^{2}+c_{\nu}|z|^{4}
$$


gives

$$
\sup _{0 \leq t \leq T}\left|v^{M}(t)\right|^{2} \leq e^{c_{\nu} T\|z\|_{C([0, T] ; H)}^{2}}\left(\left|v^{M}(0)\right|^{2}+c_{\nu} T\|z\|_{C([0, T] ; H)}^{4}\right)
$$

and integrating in time (18)

$$
v \int_{0}^{T}\left\|v^{M}(s)\right\|_{1}^{2} d s \leq\left|v^{M}(0)\right|^{2}+T c_{\nu}\left(\|z\|_{C([0, T] ; H)}^{2}\left\|v^{M}\right\|_{C([0, T] ; H)}^{2}+\|z\|_{C([0, T] ; H)}^{4}\right) .
$$

Moreover, when $\alpha \geq 0$ we proceed in a similar way: we take the scalar product of the left hand side of Eq. (17) with $A^{\alpha} v^{M}$ in $H$; then

$$
\begin{aligned}
\frac{1}{2} \frac{d}{d t}\left\|v^{M}\right\|_{\alpha}^{2}+v\left\|v^{M}\right\|_{1+\alpha}^{2} & =-\left(A^{\frac{-1+\alpha}{2}} B^{M}\left(v^{M}+z^{M}, v^{M}+z^{M}\right), A^{\frac{1+\alpha}{2}} v^{M}\right) \\
& \leq\left\|B^{M}\left(v^{M}+z^{M}, v^{M}+z^{M}\right)\right\|_{-1+\alpha}\left\|v^{M}\right\|_{1+\alpha} \\
& \leq c\left|v^{M}+z^{M}\right|\left\|v^{M}+z^{M}\right\|_{\alpha}\left\|v^{M}\right\|_{1+\alpha} \text { by (7) } \\
& \leq \frac{v}{2}\left\|v^{M}\right\|_{1+\alpha}^{2}+\frac{c_{v}}{2}\left(\left|v^{M}\right|^{2}+\left|z^{M}\right|^{2}\right)\left\|v^{M}\right\|_{\alpha}^{2}+\frac{c_{v}}{2}\left\|z^{M}\right\|_{\alpha}^{4}
\end{aligned}
$$

This gives

$$
\frac{d}{d t}\left\|v^{M}\right\|_{\alpha}^{2}+v\left\|v^{M}\right\|_{1+\alpha}^{2} \leq c_{\nu}\left(\left|v^{M}\right|^{2}+\|z\|_{\alpha}^{2}\right)\left\|v^{M}\right\|_{\alpha}^{2}+c_{\nu}\|z\|_{\alpha}^{4} .
$$

Therefore, using (19) and the fact that $\alpha \geq 0$ we get

$$
\begin{aligned}
\sup _{0 \leq t \leq T}\left\|v^{M}(t)\right\|_{\alpha}^{2} \leq & \left\|v^{M}(0)\right\|_{\alpha}^{2} e^{c_{\nu} \int_{0}^{T}\left(\left|v^{M}(t)\right|^{2}+\|z(t)\|_{\alpha}^{2}\right) d t} \\
& +c_{\nu} \int_{0}^{T} e^{c_{\nu} \int_{t}^{T}\left(\left|v^{M}(s)\right|^{2}+\|z(s)\|_{\alpha}^{2}\right) d s}\|z(t)\|_{\alpha}^{4} d t
\end{aligned}
$$

and integrating in time (21) we get a similar estimate for $\int_{0}^{T}\left\|v^{M}(s)\right\|_{1+\alpha}^{2} d s$.

Summing up, we have

$$
\begin{aligned}
& \sup _{M}\left\|v^{M}\right\|_{L^{\infty}\left(0, T ; H^{\alpha}\right)}^{2}<\infty, \\
& \sup _{M}\left\|v^{M}\right\|_{L^{2}\left(0, T ; H^{1+\alpha}\right)}^{2}<\infty .
\end{aligned}
$$

In addition $\frac{d v^{M}}{d t}$ is bounded: indeed

$$
\frac{d v^{M}}{d t}(t)=-v A v^{M}(t)-B\left(v^{M}(t)+z^{M}(t), v^{M}(t)+z^{M}(t)\right) ;
$$

using (24)-(23), we have that the first term in the r.h.s. belongs to the space $L^{2}\left(0, T ; H^{\alpha-1}\right)$ and the second to the space $C\left([0, T] ; H^{\alpha-1}\right)$ (use (11)) and thus in $L^{2}\left(0, T ; H^{\alpha-1}\right)$. Then

$$
\sup _{M}\left\|\frac{d v^{M}}{d t}\right\|_{L^{2}\left(0, T ; H^{\alpha-1}\right)}^{2}<\infty .
$$


Since the space $\left\{v: v \in L^{2}\left(0, T ; H^{1+\alpha}\right), \frac{d v}{d t} \in L^{2}\left(0, T ; H^{\alpha-1}\right)\right\}$ is compactly embedded in the space $L^{2}\left(0, T ; H^{\alpha}\right)$, from (23)-(25) we get that there exists a subsequence $\left\{v^{M_{i}}\right\}$ weakly convergent to a $v$ in $L^{2}\left(0, T ; H^{1+\alpha}\right)$, weakly-* convergent in $L^{\infty}\left(0, T ; H^{\alpha}\right)$ and strongly convergent in $L^{2}\left(0, T ; H^{\alpha}\right)$. By means of the bilinearity of $B$, of the strong convergence result and of (12), we conclude that the limit $v$ fulfills (16).

The fact that $v \in C\left([0, T] ; H^{\alpha}\right)$ comes from a result in Temam [27] (Lemma 1.4, page 263): if $v \in L^{2}\left(0, T ; H^{1+\alpha}\right)$ and $\frac{d v}{d t} \in L^{2}\left(0, T ; H^{-1+\alpha}\right)$, then $v \in C\left([0, T] ; H^{\alpha}\right)$.

Remark 3.3 We can prove also the uniqueness of this solution $v$, but we do not need it here. Anyway, the proof of uniqueness would be based on the same estimates as in the next Sect. 3.1.2.

We conclude for $u=v+z$.

Proposition 3.4 We consider the same assumptions as in Theorem 3.1. Let $u(0) \in H^{\alpha}$. Then there exists a solution to Eq. (13) such that

$$
u \in C\left([0, T] ; H^{\alpha}\right) \quad \mathbb{P} \text {-a.s. }
$$

\subsubsection{Pathwise Uniqueness}

Now we prove that the strong solution $u$ constructed in the previous section is pathwise unique, that is

Proposition 3.5 We consider the same assumptions as in Theorem 3.1. Let $u_{1}, u_{2}$ be two solutions to Eq. (13) with the same initial data, defined on the same stochastic basis and with the same Wiener process. Then $u_{1}=u_{2} \mathbb{P}$-a.s., the equality being in $C\left([0, T] ; H^{\alpha}\right)$.

Proof We proceed pathwise. Let $u_{1}, u_{2} \in C\left([0, T] ; H^{\alpha}\right)$ be two paths (in a set of $\mathbb{P}$-measure 1).

Set $U=u_{1}-u_{2}$. Then $U \in C\left([0, T] ; H^{\alpha}\right)$ and it solves an equation which is deterministic (for any path):

$$
\frac{d U}{d t}+v A U+B\left(u_{1}, u_{1}\right)-B\left(u_{2}, u_{2}\right)=0 ; \quad U(0)=0 .
$$

First, we notice that $U$ is more regular than the $u_{i}$ 's (the noise term has disappeared and we expect more regularity as for Eq. (16)).

By the bilinearity of the operator $B$, we have

$$
\frac{d U}{d t}+v A U+B\left(u_{1}, U\right)+B\left(U, u_{2}\right)=0 ; \quad U(0)=0 .
$$

We get an a priori estimate:

$$
\begin{aligned}
& \frac{1}{2} \frac{d}{d t}\|U(t)\|_{\alpha}^{2}+v\|U(t)\|_{1+\alpha}^{2} \\
& \quad=-\left(A^{\frac{\alpha-1}{2}}\left[B\left(u_{1}(t), U(t)\right)+B\left(U(t), u_{2}(t)\right)\right], A^{\frac{\alpha+1}{2}} U(t)\right) \\
& \quad \leq\left[\left\|u_{1}(t)\right\|_{\alpha}+\left\|u_{2}(t)\right\|_{\alpha}\right]\|U(t)\|_{\alpha}\|U(t)\|_{1+\alpha} \quad \text { by }(6)
\end{aligned}
$$




$$
\leq \frac{v}{2}\|U(t)\|_{1+\alpha}^{2}+\frac{c_{v}}{2}\left[\left\|u_{1}(t)\right\|_{\alpha}^{2}+\left\|u_{2}(t)\right\|_{\alpha}^{2}\right]\|U(t)\|_{\alpha}^{2} .
$$

Therefore

$$
\frac{d}{d t}\|U(t)\|_{\alpha}^{2} \leq c_{\nu}\left[\left\|u_{1}(t)\right\|_{\alpha}^{2}+\left\|u_{2}(t)\right\|_{\alpha}^{2}\right]\|U(t)\|_{\alpha}^{2} ;
$$

using Gronwall inequality it follows

$$
\|U(t)\|_{\alpha}^{2} \leq\|U(0)\|_{\alpha}^{2} e^{c_{\nu} \int_{0}^{t}\left[\left\|u_{1}(s)\right\|_{\alpha}^{2}+\left\|u_{2}(s)\right\|_{\alpha}^{2}\right] d s} .
$$

Finally, $U(t)=0$ for all $t$, since $U(0)=0$.

Remark 3.6 Markovianity is inherited from the Galerkin approximations.

\subsubsection{Feller Property}

Let us denote by $u(t ; x)$ the solution of Eq. (13) with initial data $x$, by $B_{b}\left(H^{\alpha}\right)$ the space of Borel bounded functions $\phi: H^{\alpha} \rightarrow \mathbb{R}$ and by $C_{b}\left(H^{\alpha}\right)$ its subspace of continuous bounded functions.

Define the Markov semigroup $P_{t}: B_{b}\left(H^{\alpha}\right) \rightarrow B_{b}\left(H^{\alpha}\right)$ as

$$
P_{t} \phi(x)=\mathbb{E}[\phi(u(t ; x))] .
$$

This is a contraction semigroup. Moreover, it is Feller in $H^{\alpha}$, that is

$$
P_{t}: C_{b}\left(H^{\alpha}\right) \rightarrow C_{b}\left(H^{\alpha}\right) .
$$

This comes from the estimates for the pathwise uniqueness. Indeed, (28) gives

$$
\|u(t ; x)-u(t ; y)\|_{\alpha}^{2} \leq\|x-y\|_{\alpha}^{2} e^{c_{\nu} \int_{0}^{t}\left[\|u(s ; x)\|_{\alpha}^{2}+\|u(s ; y)\|_{\alpha}^{2}\right] d s}
$$

for $t>0$ fixed. By (22) we get a uniform estimate of $\|u(\cdot ; x)\|_{L^{\infty}\left(0, T ; H^{\alpha}\right)}^{2}$ when $\|x\|_{\alpha}$ is bounded, i.e.

$$
\forall R>0 \exists C_{R}: \quad \sup _{\|x\|_{\alpha} \leq R}\|u(\cdot ; x)\|_{C\left([0, T] ; H^{\alpha}\right)}<C_{R} .
$$

Hence, when $\|x-y\|_{\alpha} \rightarrow 0$ from (29) and (30) we get $\|u(t ; x)-u(t ; y)\|_{\alpha} \rightarrow 0$. We conclude that $\phi(u(t ; x)) \rightarrow \phi(u(t ; y))$ for $\phi \in C_{b}\left(H^{\alpha}\right)$ and therefore $\mathbb{E}[\phi(u(t ; x))] \rightarrow$ $\mathbb{E}[\phi(u(t ; y))]$ by the dominated convergence. This means that $P_{t} \phi \in C_{b}\left(H^{\alpha}\right)$ for any $t>0$ and $\phi \in C_{b}\left(H^{\alpha}\right)$.

\subsection{Invariant Measure}

We prove the following theorem:

Theorem 3.7 Besides the assumptions of Theorem 3.1 we consider (C3). Then, $\mu^{\beta, v}$ is the unique invariant measure for Eq. (13), in the sense that

$$
\int P_{t} \phi d \mu^{\beta, v}=\int \phi d \mu^{\beta, \nu} \quad \forall \phi \in \mathcal{L}^{1}\left(\mu^{\beta, \nu}\right) \text { and } t \geq 0 .
$$


First, we show that $\mu^{\beta, \nu}$ is an invariant measure for the nonlinear equation (13) in the sense that

$$
\int P_{t} \phi d \mu^{\beta, v}=\int \phi d \mu^{\beta, v} \quad \forall \phi \in C_{b}\left(H^{\alpha}\right) \text { and } t \geq 0 .
$$

Then, we prove the same relationship for all $\phi \in \mathcal{L}^{1}\left(\mu^{\beta, \nu}\right)$ by extending $P_{t}$ from $C_{b}$ to $\mathcal{L}^{1}\left(\mu^{\beta, \nu}\right)$. The next step is to prove the uniqueness of the invariant measure.

A consequence of this result is the following

Corollary 3.8 Given any initial data with law $\mu^{\beta, \nu}$, there exists a unique stationary solution of Eq. (13) whose law at any fixed time is $\mu^{\beta, \nu}$.

Finally, in Sect. 3.2.4 we analyze the rate of convergence of $P_{t} \phi$, as $t \rightarrow \infty$.

\subsubsection{Existence of the Invariant Measure}

We know that the linear stochastic equation (15) has $\mu^{\beta, v}$ as unique invariant measure, that is $\mu^{\beta, \nu}$ is the unique probability measure such that

$$
\int \mathbb{E}[\phi(z(t ; x))] \mu^{\beta, v}(d x)=\int \phi(x) \mu^{\beta, v}(d x) \quad \forall t \geq 0, \phi \in B_{b}\left(H^{\alpha}\right)
$$

(see $[14,15])$. Actually we can define the latter relationship for all $\phi \in \mathcal{L}^{p}\left(\mu^{\beta, \nu}\right)$, given any $1 \leq p<\infty$ (see, e.g., [11, 12]).

Now, we want to show that $\mu^{\beta, v}$ is an invariant measure also for the nonlinear equation (13).

Following the lines of [4], we define the measure $\mu_{M}^{\beta, v}=\bigotimes_{j=1}^{M} \mathcal{N}\left(0, \frac{1}{v} \lambda_{j}^{-\beta}\right)$ on Borel subsets of $\mathbb{R}^{M}$. In a standard way, taking as initial value $u^{M}(0)$ a random variable of law $\mu_{M}^{\beta, v}$ then the Galerkin system

$$
d u^{M}(t)+\left[v A u^{M}(t)+B^{M}\left(u^{M}(t), u^{M}(t)\right)\right] d t=\Pi_{M} \sqrt{2 A^{1-\beta}} d w(t)
$$

has a unique strong $\mu_{M}^{\beta, v}$-stationary solution; the important point is that $\mu_{M}^{\beta, v}$ is an invariant measure for (32) (see Sect. 3.2 in [4]). Thus, the infinite dimensional equation

$$
d U^{M}(t)+\left[v A U^{M}(t)+B^{M}\left(U^{M}(t), U^{M}(t)\right)\right] d t=\sqrt{2 A^{1-\beta}} d w(t)
$$

has a $\mu^{\beta, v}$-stationary solution (notice that $U^{M}=u^{M}+\left(I-\Pi_{M}\right) z$ ). Passing to the limit as $M \rightarrow \infty$ we get that also Eq. (13) with initial data of law $\mu^{\beta, v}$ has a $\mu^{\beta, \nu}$-stationary solution, that is

$$
\int P_{t} \phi d \mu^{\beta, v}=\int \phi d \mu^{\beta, v} \quad \forall t \geq 0, \phi \in C_{b}\left(H^{\alpha}\right) .
$$

\subsubsection{Extension of $P_{t}$}

In this section, we assume (C3). Now we want to improve (34) considering $\phi \in \mathcal{L}^{1}\left(\mu^{\beta, \nu}\right)$. To this end, we use an approximative criterium of Eberle [17]. The result of this section will be useful to prove results in Sect. 3.2.4.

We introduce the Kolmogorov operator associated to the stochastic equation (13). Let $F C_{b}^{\infty}$ be the space of infinitely differentiable cylindrical functions bounded and with 
bounded derivatives; $\phi \in F C_{b}^{\infty}$ means that there exist $m \in \mathbb{N}, \tilde{\phi} \in C_{b}^{\infty}\left(\mathbb{R}^{m}\right)$ and multiindices $\left(i_{1}, i_{2}, \ldots, i_{m}\right)$ such that

$$
\phi(x)=\tilde{\phi}\left(\left(x, e_{i_{1}}\right),\left(x, e_{i_{2}}\right), \ldots,\left(x, e_{i_{m}}\right)\right) .
$$

We set $\frac{\partial \phi}{\partial x_{i}}=\frac{\partial \tilde{\phi}}{\partial x_{i}}$ with $x_{i}=\left(x, e_{i}\right) . F C_{b}^{\infty}$ is a dense subset of $\mathcal{L}^{p}\left(\mu^{\beta, v}\right)$ for any $p \geq 1$.

We define the Kolmogorov operator first on these very regular functions $\phi \in F C_{b}^{\infty}$ as

$$
K \phi(x)=\sum_{j}\left[\lambda_{j}^{1-\beta} \frac{\partial^{2} \phi}{\partial x_{j}^{2}}(x)-B_{j}(x, x) \frac{\partial \phi}{\partial x_{j}}(x)-v \lambda_{j} x_{j} \frac{\partial \phi}{\partial x_{j}}(x)\right] .
$$

We have that $K \phi \in \mathcal{L}^{1}\left(\mu^{\beta, \nu}\right)$ for any $\phi \in F C_{b}^{\infty}$ (use that, thanks to (4), each $B_{j} \in \mathcal{L}^{1}\left(\mu^{\beta, v}\right)$ and the sums are finite).

First, we prove that $\mu^{\beta, v}$ is an infinitesimally invariant measure for Eq. (13) in the sense that

$$
\int K \phi d \mu^{\beta, v}=0 \quad \forall \phi \in F C_{b}^{\infty} .
$$

Indeed, we can write $K$ as the sum of two operators, $K=Q+L$, with domains $F C_{b}^{\infty}$ and we have the infinitesimal invariance for both these operators. We integrate by parts:

$$
\int Q \phi d \mu^{\beta, v} \equiv \int \sum_{j}\left[\lambda_{j}^{1-\beta} \frac{\partial^{2} \phi}{\partial x_{j}^{2}}(x)-v \lambda_{j} x_{j} \frac{\partial \phi}{\partial x_{j}}(x)\right] \mu^{\beta, v}(d x)=0
$$

and

$$
\begin{aligned}
\int L \phi d \mu^{\beta, v} & \equiv-\int \sum_{j} B_{j}(x, x) \frac{\partial \phi}{\partial x_{j}}(x) \mu^{\beta, v}(d x) \\
& =-v \int \underbrace{\sum_{j} \lambda_{j}^{\beta} B_{j}(x, x) x_{j}}_{=0 \text { by }(\mathrm{C} 2 \text { ii })} \phi(x) \mu^{\beta, v}(d x)=0 .
\end{aligned}
$$

We have used that each $B_{j}$ does not depend on the variable $x_{j}$.

Moreover, (36) implies that the Kolmogorov operator $\left(K, F C_{b}^{\infty}\right)$ is dissipative on $\mathcal{L}^{p}\left(\mu^{\beta, \nu}\right)$ for any $1 \leq p<\infty$ (see Lemma 1.8 in [17]). Hence it is closable in $\mathcal{L}^{p}\left(\mu^{\beta, v}\right)$ (see [26]).

Now we have

Proposition 3.9 Besides the assumptions of Theorem 3.1 we consider (C3). Then, the closure operator $\bar{K}$ of the Kolmogorov operator $\left(K, F C_{b}^{\infty}\right)$ in $\mathcal{L}^{1}\left(\mu^{\beta, v}\right)$ generates a subMarkovian strongly continuous semigroup $T_{t}=e^{\bar{K} t}$ in $\mathcal{L}^{1}\left(\mu^{\beta, v}\right)$.

Moreover, $T_{t}$ is the only strongly continuous semigroup on $\mathcal{L}^{1}\left(\mu^{\beta, v}\right)$ which has generator that extends $\left(K, F C_{b}^{\infty}\right)$ (see Appendix $\mathrm{A}$ in [17]).

We postpone the proof of this result and continue our analysis. Since $F C_{b}^{\infty}$ is a core for the infinitesimal generator of $T_{t}$ in $\mathcal{L}^{1}\left(\mu^{\beta, \nu}\right)$ by density from (36) we get that

$$
\int \bar{K} \phi d \mu^{\beta, v}=0 \quad \forall \phi \in D(\bar{K}) .
$$


This is equivalent to

$$
\int T_{t} \phi d \mu^{\beta, v}=\int \phi d \mu^{\beta, \nu} \quad \forall \phi \in \mathcal{L}^{1}\left(\mu^{\beta, \nu}\right) \text { and } t \geq 0
$$

The semigroup $\left\{T_{t}\right\}_{t \geq 0}$ provides a martingale $\mu^{\beta, v}$-stationary solution to the stochastic equation (13) (see, e.g., [17] and references therein). Therefore $\left\{T_{t}\right\}$ is an extension of $\left\{P_{t}\right\}$ on $\mathcal{L}^{1}\left(\mu^{\beta, \nu}\right)$. Henceforth, we denote these semigroups in $C_{b}\left(H^{\alpha}\right)$ and $\mathcal{L}^{1}\left(\mu^{\beta, \nu}\right)$ with the same symbol $P_{t}$. Finally, (39) means that (31) holds true.

Now, we go back to the proof of Proposition 3.9. We refer to [17] for all the details; in particular, we use Theorem 5.2, Corollary 5.3, Lemma 5.11 and (5.46) at page 226 of [17] with $p=1$.

Proof of Proposition 3.9 From Lumer-Phillips theorem we know that the closure of the operator $\left(K, F C_{b}^{\infty}\right)$ in $\mathcal{L}^{1}\left(\mu^{\beta, \nu}\right)$ generates a strongly continuous semigroup $T_{t}$ if and only if the range of $\left(\lambda-K, F C_{b}^{\infty}\right)$ is dense in $\mathcal{L}^{1}\left(\mu^{\beta, v}\right)$ for some (and all) $\lambda>0$. To prove the density result, we use an approximative criterium:

$$
\forall F \in \mathcal{L}^{1}\left(\mu^{\beta, \nu}\right) \forall \varepsilon>0 \exists v \in F C_{b}^{\infty}: \quad\|(\lambda-K) v-F\|_{\mathcal{L}^{1}\left(\mu^{\beta, \nu}\right)}<\varepsilon .
$$

Now, we take $F \in \mathcal{L}^{1}\left(\mu^{\beta, \nu}\right)$. Then there exists a sequence $\left\{F^{N}\right\}_{N \in \mathbb{N}}$ with $F^{N} \in C_{b}^{\infty}\left(\mathbb{R}^{N}\right)$ and

$$
\lim _{N \rightarrow \infty}\left\|F^{N}-F\right\|_{\mathcal{L}^{1}\left(\mu^{\beta, \nu}\right)}=0, \quad \sup _{N}\left\|F^{N}\right\|_{C_{b}}<\infty .
$$

On the other hand, the assumption $B_{n} \in \mathcal{L}^{2}\left(\mu^{\beta, \nu}\right)$ (for any $n$ ) implies that $B^{N} \in \mathcal{L}^{2}\left(\mu^{\beta, v}\right)$ for any $N$, and therefore there exists a sequence $\left\{C^{N}\right\}_{N \in \mathbb{N}}$ with $C^{N} \in C_{b}^{\infty}\left(\mathbb{R}^{N} \rightarrow \mathbb{R}^{N}\right)$ and

$$
\left\|B^{N}-C^{N}\right\|_{\mathcal{L}^{2}\left(\mu^{\beta, \nu}\right)} \leq \frac{1}{N} .
$$

Bearing in mind (5), this implies that

$$
\left\|\Pi_{N} B-C^{N}\right\|_{\mathcal{L}^{2}\left(\mu^{\beta, \nu}\right)} \leq\left\|\Pi_{N} B-B^{N}\right\|_{\mathcal{L}^{2}\left(\mu^{\beta, v}\right)}+\left\|B^{N}-C^{N}\right\|_{\mathcal{L}^{2}\left(\mu^{\beta, \nu}\right)} \rightarrow 0
$$

as $N \rightarrow \infty$.

For each $N$, we introduce a regularized finite dimensional Kolmogorov operator $K^{N}$ acting on functions $\phi \in C_{b}^{\infty}\left(\mathbb{R}^{N}\right)$ :

$$
\left(K^{N} \phi\right)(x)=\sum_{j=1}^{N}\left[\lambda_{j}^{1-\beta} \frac{\partial^{2} \phi}{\partial x_{j}^{2}}(x)-C_{j}^{N}(x) \frac{\partial \phi}{\partial x_{j}}(x)-v \lambda_{j} x_{j} \frac{\partial \phi}{\partial x_{j}}(x)\right] .
$$

It has smooth coefficients. Therefore, given $F^{N} \in C_{b}^{\infty}\left(\mathbb{R}^{N}\right)$ and $\lambda>0$ the equation

$$
\left(\lambda-K^{N}\right) \phi^{N}=F^{N}
$$

has a unique solution $\phi^{N} \in C_{b}^{\infty}\left(\mathbb{R}^{N}\right)$; moreover

$$
\lambda\left\|\phi^{N}\right\|_{C_{b}} \leq\left\|F^{N}\right\|_{C_{b}} .
$$


Further, setting $\left|\sqrt{A^{\gamma}} D \phi^{N}\right|^{2}=\sum_{j=1}^{N} \lambda_{j}^{\gamma}\left|\frac{\partial \phi^{N}}{\partial x_{j}}\right|^{2}$, by a straightforward computation we have

$$
K\left(\phi^{N}\right)^{2}=2 \phi^{N} K \phi^{N}+2\left|\sqrt{A^{1-\beta}} D \phi^{N}\right|^{2} .
$$

Using the infinitesimal invariance (36), we have

$$
\begin{aligned}
\left\|\sqrt{A^{1-\beta}} D \phi^{N}\right\|_{\mathcal{L}^{2}\left(\mu^{\beta, v}\right)}^{2}= & -\int \phi^{N} K \phi^{N} d \mu^{\beta, \nu} \\
= & -\int \phi^{N}\left[K \phi^{N}-K^{N} \phi^{N}\right] d \mu^{\beta, v}-\int \phi^{N} K^{N} \phi^{N} d \mu^{\beta, v} \\
= & -\int \phi^{N} \sum_{j=1}^{N}\left(B_{j}-C_{j}^{N}\right) \frac{\partial \phi^{N}}{\partial x_{j}} d \mu^{\beta, v}+\int \phi^{N}\left(F^{N}-\lambda \phi^{N}\right) d \mu^{\beta, v} \\
\leq & \left\|\phi^{N}\right\|_{C_{b}}\left\|\Pi_{N} B-C^{N}\right\|_{\mathcal{L}^{2}\left(\mu^{\beta, v}\right)}\left\|D \phi^{N}\right\|_{\mathcal{L}^{2}\left(\mu^{\beta, \nu}\right)} \\
& +\left\|\phi^{N}\right\|_{C_{b}}\left(\left\|F^{N}\right\|_{C_{b}}+\lambda\left\|\phi^{N}\right\|_{C_{b}}\right) .
\end{aligned}
$$

Using (45) and the fact that $\lambda_{1}^{1-\beta}\left\|D \phi^{N}\right\|_{\mathcal{L}^{2}\left(\mu^{\beta, \nu}\right)}^{2} \leq\left\|\sqrt{A^{1-\beta}} D \phi^{N}\right\|_{\mathcal{L}^{2}\left(\mu^{\beta, \nu}\right)}^{2}$ for $\beta \leq 1$, we get that there exists a constant $C_{\beta, \lambda}>0$ such that

$$
\left\|D \phi^{N}\right\|_{\mathcal{L}^{2}\left(\mu^{\beta, v}\right)} \leq C_{\beta, \lambda}\left\|F^{N}\right\|_{C_{b}}\left[1+\left\|\Pi_{N} B-C^{N}\right\|_{\mathcal{L}^{2}\left(\mu^{\beta, \nu}\right)}\right]
$$

Let us go back to (40); by (44) we have

$$
(\lambda-K) \phi^{N}-F=\left(K^{N}-K\right) \phi^{N}+F^{N}-F \equiv \sum_{j=1}^{N}\left[B_{j}-C_{j}^{N}\right] \frac{\partial \phi^{N}}{\partial x_{j}}+F^{N}-F .
$$

Integrating with respect to the measure $\mu^{\beta, v}$ we get

$$
\begin{aligned}
\left\|(\lambda-K) \phi^{N}-F\right\|_{\mathcal{L}^{1}\left(\mu^{\beta, \nu}\right)} & \leq \int\left|\sum_{j=1}^{N}\left[B_{j}-C_{j}^{N}\right] \frac{\partial \phi^{N}}{\partial x_{j}}\right| d \mu^{\beta, v}+\left\|F^{N}-F\right\|_{\mathcal{L}^{1}\left(\mu^{\beta, v}\right)} \\
& \leq\left\|\Pi_{N} B-C^{N}\right\|_{\mathcal{L}^{2}\left(\mu^{\beta, v}\right)}\left\|D \phi^{N}\right\|_{\mathcal{L}^{2}\left(\mu^{\beta, v}\right)}+\left\|F^{N}-F\right\|_{\mathcal{L}^{1}\left(\mu^{\beta, v}\right)}
\end{aligned}
$$

by Schwarz inequality. Using (46) we find

$$
\begin{aligned}
\left\|(\lambda-K) \phi^{N}-F\right\|_{\mathcal{L}^{1}\left(\mu^{\beta, \nu}\right)} \leq & C_{\beta, \lambda}\left\|\Pi_{N} B-C^{N}\right\|_{\mathcal{L}^{2}\left(\mu^{\beta, \nu}\right)}\left\|F^{N}\right\|_{C_{b}}\left[1+\left\|\Pi_{N} B-C^{N}\right\|_{\mathcal{L}^{2}\left(\mu^{\beta, \nu}\right)}\right] \\
& +\left\|F^{N}-F\right\|_{\mathcal{L}^{1}\left(\mu^{\beta, \nu}\right)} .
\end{aligned}
$$

Bearing in mind the assumptions on the approximating terms and (43), we find (40).

Remark 3.10 Because of the invariance of the measure $\mu^{\beta, \nu}$, the contraction semigroup $P_{t}$ in $C_{b}\left(H^{\alpha}\right)$ can be uniquely extended to a strongly continuous contraction semigroup in $\mathcal{L}^{p}\left(\mu^{\beta, v}\right)$ also for any $p>1$. Indeed,

$$
\left|P_{t} \phi(x)\right|^{p}=|\mathbb{E}[\phi(u(t ; x))]|^{p} \leq \mathbb{E}\left[|\phi(u(t ; x))|^{p}\right]=P_{t}|\phi|^{p}(x)
$$


and by the invariance of the measure $\mu^{\beta, \nu}$

$$
\int\left|P_{t} \phi\right|^{p} d \mu^{\beta, \nu} \leq \int P_{t}|\phi|^{p} d \mu^{\beta, \nu}=\int|\phi|^{p} d \mu^{\beta, \nu}
$$

Since $C_{b}\left(H^{\alpha}\right)$ is dense in $\mathcal{L}^{p}\left(\mu^{\beta, \nu}\right)$, we can uniquely define the semigroup on $\mathcal{L}^{p}\left(\mu^{\beta, \nu}\right)$ for any $p>1$. We use the same symbol $P_{t}$ to denote all these semigroups.

Notice that in condition (C3) we require $\int\left|B_{n}(x, x)\right|^{2} \mu^{\beta, v}(d x)<\infty$ for any $n$. Therefore $K: F C_{b}^{\infty} \rightarrow \mathcal{L}^{2}\left(\mu^{\beta, \nu}\right)$. Moreover, according to Corollary 5.3 of [17], we have that the restriction of $T_{t}$ to $\mathcal{L}^{2}\left(\mu^{\beta, \nu}\right)$ is a strongly continuous semigroup on $\mathcal{L}^{2}\left(\mu^{\beta, \nu}\right)$ and the generator of this semigroup again extends $\left(K, F C_{b}^{\infty}\right)$. In the sequel we will use the same symbol to denote these semigroups in both spaces $\mathcal{L}^{1}\left(\mu^{\beta, \nu}\right)$ and $\mathcal{L}^{2}\left(\mu^{\beta, \nu}\right)$.

\subsubsection{Uniqueness of the Invariant Measure}

Now we prove that Eq. (13) has at most one invariant measure. We use the results of Sect. 3.1.

Proposition 3.11 With the assumptions of Theorem 3.1, there exists at most one invariant measure for Eq. (13).

Proof Let $R(t, x, \cdot)$ be the law of $z(t ; x)$ and $P(t, x, \cdot)$ be the law of $u(t ; x)$. Then any $R(t, x, \cdot)$ is equivalent to the Gibbs measure $\mu^{\beta, v}$ (see, e.g., [15]); we write it as $R(t, x, \cdot) \sim$ $\mu^{\beta, v}$. Moreover we have that

$$
\int_{0}^{T}\left|\sqrt{A^{\beta-1}} B(z(t), z(t))\right|^{2} d t<\infty \quad \mathbb{P} \text {-a.s. }
$$

and

$$
\int_{0}^{T}\left|\sqrt{A^{\beta-1}} B(u(t), u(t))\right|^{2} d t<\infty \quad \mathbb{P} \text {-a.s. }
$$

For this use that $\|B(x, x)\|_{\beta-1} \leq c\|x\|_{\alpha}^{2}$ from assumption (6) and that $\mathbb{P}\left\{z \in C\left([0, T] ; H^{\alpha}\right)\right\}=\mathbb{P}\left\{u \in C\left([0, T] ; H^{\alpha}\right)\right\}=1$.

According to Theorem 9.2 in [18] (see also [19]), (47)-(48) imply that the measure $P(t, x, \cdot)$ is equivalent to $R(t, x, \cdot)$. On the other side $R(t, x, \cdot) \sim R(s, y, \cdot) \sim \mu^{\beta, \nu}$, hence we get

$$
P(t, x, \cdot) \sim P(t, y, \cdot) \sim \mu^{\beta, v}
$$

for any $x, y \in H^{\alpha}$ and $t>0$. Using Doob theorem (see, e.g., Theorem 4.2.1 in [15]), we deduce that there exists at most one invariant measure.

By means of the existence result of the previous section, we get that $\mu^{\beta, v}$ is the unique invariant measure for Eq. (13). Moreover, it is strongly mixing

$$
\lim _{t \rightarrow \infty} P(t, x, \Gamma)=\mu^{\beta, v}(\Gamma)
$$

for arbitrary $x \in H^{\alpha}$ and Borel set $\Gamma$ in $H^{\alpha}$. 


\subsubsection{Rate of Convergence}

Now, we consider the semigroup $P_{t}$ in $\mathcal{L}^{2}\left(\mu^{\beta, \nu}\right)$ (see Remark 3.10).

We recall the "Carré du champ" identity. For the reader's convenience we give the proof (see, e.g., [11]).

Proposition 3.12 Besides the assumptions of Theorem 3.1 we consider (C3). Then, we have

$$
\int \phi \bar{K} \phi d \mu^{\beta, v}=-\int\left|\sqrt{A^{1-\beta}} D \phi\right|^{2} d \mu^{\beta, \nu} \quad \forall \phi \in D(\bar{K}) .
$$

Proof First we take $\phi \in F C_{b}^{\infty}$. A straightforward computation yields that

$$
K \phi^{2}=2 \phi K \phi+2\left|\sqrt{A^{1-\beta}} D \phi\right|^{2} .
$$

By the $\mu^{\beta, \nu}$-infinitesimal invariance, we have $\int K \phi^{2} d \mu^{\beta, v}=0$; thus

$$
\int \phi K \phi d \mu^{\beta, v}=-\int\left|\sqrt{A^{1-\beta}} D \phi\right|^{2} d \mu^{\beta, v}
$$

Now, taking $\phi \in D(\bar{K})$, we use that $F C_{b}^{\infty}$ is a core for $\bar{K}$; therefore there exists a sequence $\left\{\phi_{n}\right\} \subset F C_{b}^{\infty}$ such that

$$
\phi_{n} \rightarrow \phi, \quad K \phi_{n} \rightarrow \bar{K} \phi \quad \text { in } \mathcal{L}^{2}\left(\mu^{\beta, \nu}\right) .
$$

From (51) we get

$$
\int\left|\sqrt{A^{1-\beta}} D\left(\phi_{n}-\phi_{m}\right)\right|^{2} d \mu^{\beta, v} \leq \int\left|\phi_{n}-\phi_{m}\right|\left|K\left(\phi_{n}-\phi_{m}\right)\right| d \mu^{\beta, v}
$$

Hence, the sequence $\left\{\sqrt{A^{1-\beta}} D \phi_{n}\right\}$ is a Cauchy sequence in $\mathcal{L}^{2}\left(\mu^{\beta, v}\right)$ and we get (50).

Now, given $\phi \in \mathcal{L}^{2}\left(\mu^{\beta, v}\right)$ we set $\bar{\phi}=\int \phi d \mu^{\beta, \nu}$; then we have the following theorem on the rate of convergence of $P_{t} \phi$ as $t \rightarrow \infty$.

Theorem 3.13 Besides the assumptions of Theorem 3.1 we consider (C3). Then

$$
\int\left|P_{t} \phi(x)-\bar{\phi}\right|^{2} \mu^{\beta, v}(d x) \leq e^{-\lambda_{1} t} \int|\phi(x)-\bar{\phi}|^{2} \mu^{\beta, v}(d x)
$$

for any $\phi \in \mathcal{L}^{2}\left(\mu^{\beta, \nu}\right)$ and $t>0$.

Proof Let us define the space

$$
\mathcal{L}_{0}^{2}\left(\mu^{\beta, v}\right)=\left\{\phi \in \mathcal{L}^{2}\left(\mu^{\beta, v}\right): \bar{\phi}=0\right\} ;
$$

it is not difficult to prove that it is invariant for the semigroup $P_{t}$ (see [12]).

First, let us take $\phi \in \mathcal{L}_{0}^{2}\left(\mu^{\beta, v}\right) \cap D(\bar{K})$; then $P_{t} \phi \in \mathcal{L}_{0}^{2}\left(\mu^{\beta, v}\right) \cap D(\bar{K})$ and by the HilleYosida theorem

$$
\frac{d}{d t} P_{t} \phi=\bar{K} P_{t} \phi
$$


Therefore, bearing in mind (50)

$$
\frac{1}{2} \frac{d}{d t} \int\left|P_{t} \phi\right|^{2} d \mu^{\beta, \nu}=\int P_{t} \phi \bar{K} P_{t} \phi d \mu^{\beta, \nu}=-\int\left|\sqrt{A^{1-\beta}} D_{x} P_{t} \phi\right|^{2} d \mu^{\beta, \nu} .
$$

Since a Gaussian measure fulfills the spectral gap inequality (see [8]) we have

$$
\int\left|\sqrt{A^{1-\beta}} D_{x} P_{t} \phi(x)\right|^{2} \mu^{\beta, v}(d x) \geq \frac{\lambda_{1}}{2} \int\left[P_{t} \phi(x)\right]^{2} \mu^{\beta, \nu}(d x)
$$

where $\lambda_{1}>0$ is the first eigenvalue of the operator $A$. By the two latter relationships we get

$$
\frac{d}{d t} \int\left|P_{t} \phi\right|^{2} d \mu^{\beta, \nu} \leq-\lambda_{1} \int\left|P_{t} \phi\right|^{2} d \mu^{\beta, \nu} .
$$

Hence, using Gronwall lemma, we have that for any $t>0$

$$
\int\left|P_{t} \phi\right|^{2} d \mu^{\beta, \nu} \leq e^{-\lambda_{1} t} \int|\phi|^{2} d \mu^{\beta, v} \quad \forall \phi \in \mathcal{L}_{0}^{2}\left(\mu^{\beta, v}\right) \cap D(\bar{K}) .
$$

Now we take $\phi \in D(\bar{K})$; replacing $\phi$ with $\phi-\bar{\phi}$ in (53), we obtain that

$$
\int\left|P_{t} \phi-\bar{\phi}\right|^{2} d \mu^{\beta, v}=\int\left|P_{t}(\phi-\bar{\phi})\right|^{2} d \mu^{\beta, \nu} \leq e^{-\lambda_{1} t} \int|\phi-\bar{\phi}|^{2} d \mu^{\beta, \nu} .
$$

Using that $D(\bar{K})$ is dense in $\mathcal{L}^{2}\left(\mu^{\beta, v}\right)$ we get the result.

\section{An Example: Shell Models of Turbulence}

Shell models of turbulence describe the evolution of complex Fourier-like components of a scalar velocity field. Here, we present the details for the SABRA shell model (see [24]), but the same results hold for the GOY shell model (see [21, 25]). In recent years, there has been an increasing interest in these fluid dynamical models, both for the deterministic and the stochastic case (see also [3, 5, 9, 10]). From the analytic point of view as well as for numerical computations, they are easier to analyze than the Navier-Stokes or Euler equations. But, they retain many important features of the true hydrodynamical models.

Instead of dealing with complex valued unknowns we deal with the real and imaginary part of each component of the scalar velocity field (for the basic settings we follow [4]); this defines a sequence $\left\{u_{n}\right\}_{n}$ with $u_{n} \in \mathbb{R}^{2}$. For $x=\left(x_{1}, x_{2}\right) \in \mathbb{R}^{2}$ we set $|x|^{2}=x_{1}^{2}+x_{2}^{2}$ and the scalar product in $\mathbb{R}^{2}$ is $x \cdot y=x_{1} y_{1}+x_{2} y_{2}$.

Then, using the notations of Sect. 2.1, we define the basic space $H$ as

$$
H=\left\{u=\left(u_{1}, u_{2}, \ldots\right) \in\left(\mathbb{R}^{2}\right)^{\infty}: \sum_{n=1}^{\infty}\left|u_{n}\right|^{2}<\infty\right\} .
$$

The basis in $H$ is given by the sequence $\left\{e_{1}^{(1)}, e_{1}^{(2)}, e_{2}^{(1)}, e_{2}^{(2)}, e_{3}^{(1)}, e_{3}^{(2)}, \ldots\right\}$ of elements of $\left(\mathbb{R}^{2}\right)^{\infty}$, where

$$
\begin{aligned}
& e_{n}^{(1)}=((0,0), \ldots,(0,0),(1,0),(0,0), \ldots) \\
& e_{n}^{(2)}=((0,0), \ldots,(0,0),(0,1),(0,0), \ldots)
\end{aligned}
$$

with the nonvanishing vectors in place $n$. 
The eigenvalues are

$$
\lambda_{n}=k_{0}^{2} \lambda^{2 n}
$$

with $\lambda>1$.

Let us check the validity of assumptions (C1)-(C5).

Inequality (3) holds with $c_{p, v}=\left(\frac{p}{e v}\right)^{p}$. We can take any $\alpha<\beta$ to fulfill (C4).

We set $k_{n}=\sqrt{\lambda}_{n}$. The bilinear term $B$ is defined by means of the components $B_{n}=$ $\left(B_{n, 1}, B_{n, 2}\right)$ as follows (see, e.g., [4]):

$$
\begin{aligned}
& B_{1,1}(u, v)=a k_{2}\left[-u_{2,2} v_{3,1}+u_{2,1} v_{3,2}\right], \\
& B_{1,2}(u, v)=-a k_{2} u_{2} \cdot v_{3}, \\
& B_{2,1}(u, v)=a k_{3}\left[-u_{3,2} v_{4,1}+u_{3,1} v_{4,2}\right]+b k_{2}\left[-u_{1,2} v_{3,1}+u_{1,1} v_{3,2}\right], \\
& B_{2,2}(u, v)=-a k_{3} u_{3} \cdot v_{4}-b k_{2} u_{1} \cdot v_{3}
\end{aligned}
$$

and for $n>2$

$$
\begin{aligned}
B_{n, 1}(u, v)= & a k_{n+1}\left[-u_{n+1,2} v_{n+2,1}+u_{n+1,1} v_{n+2,2}\right] \\
& +b k_{n}\left[-u_{n-1,2} v_{n+1,1}+u_{n-1,1} v_{n+1,2}\right] \\
& +a k_{n-1}\left[u_{n-1,2} v_{n-2,1}+u_{n-1,1} v_{n-2,2}\right] \\
& +b k_{n-1}\left[u_{n-2,2} v_{n-1,1}+u_{n-2,1} v_{n-1,2}\right], \\
B_{n, 2}(u, v)= & -a k_{n+1}\left[u_{n+1,1} v_{n+2,1}+u_{n+1,2} v_{n+2,2}\right] \\
& -b k_{n}\left[u_{n-1,1} v_{n+1,1}+u_{n-1,2} v_{n+1,2}\right] \\
& -a k_{n-1}\left[u_{n-1,1} v_{n-2,1}-u_{n-1,2} v_{n-2,2}\right] \\
& -b k_{n-1}\left[u_{n-2,1} v_{n-1,1}-u_{n-2,2} v_{n-1,2}\right]
\end{aligned}
$$

where $a$ and $b$ are real numbers such that

$$
a+b \lambda^{2 \beta}=(a+b) \lambda^{4 \beta}
$$

for some $\beta>0$, that is

$$
\lambda^{2 \beta}=-\frac{a}{a+b}
$$

(recall that $\lambda>1$ ). This condition implies (C2 ii), whereas (C2 i) holds for any real $a$ and $b$. For instance, let us check that (58) implies (C2 ii). We have

$$
\begin{aligned}
& \sum_{n=1}^{\infty} k_{n}^{2 \beta} B_{n}(u, u) \cdot u_{n} \\
& \quad=\sum_{n=1}^{\infty} k_{n}^{2 \beta}\left[B_{n, 1}(u, u) u_{n, 1}+B_{n, 2}(u, u) u_{n, 2}\right] \\
& \quad=\sum_{n=1}^{\infty}\left[a+b \lambda^{2 \beta}-(a+b) \lambda^{4 \beta}\right] \lambda k_{n}^{2 \beta+1}\left(u_{n+2} \cdot u_{n}\right)\left(u_{n+1,2}+u_{n+1,1}\right) .
\end{aligned}
$$


Relationship (5) of (C3) holds for $\beta>\frac{1}{2}$; this includes the interesting physical case of $\beta=1$ (see Sect. 2.2). Indeed, for the SABRA shell model

$$
B_{n, 1}^{M}(x, x)-B_{n, 1}(x, x)= \begin{cases}0 & \text { for } n \leq M-2, \\ -a k_{M}\left(x_{M, 1} x_{M+1,2}-x_{M, 2} x_{M+1,1}\right) & \text { for } n=M-1, \\ -a k_{M+1}\left(x_{M+1,1} x_{M+2,2}-x_{M+1,2} x_{M+2,1}\right) & \\ -b k_{M}\left(x_{M-1,1} x_{M+1,2}-x_{M-1,2} x_{M+1,1}\right) & \text { for } n=M\end{cases}
$$

and

$$
B_{n, 2}^{M}(x, x)-B_{n, 2}(x, x)= \begin{cases}0 & \text { for } n \leq M-2, \\ -a k_{M}\left(-x_{M, 1} x_{M+1,1}-x_{M, 2} x_{M+1,2}\right) & \text { for } n=M-1, \\ -a k_{M+1}\left(-x_{M+1,1} x_{M+2,1}-x_{M+1,2} x_{M+2,2}\right) & \\ -b k_{M}\left(-x_{M-1,1} x_{M+1,1}-x_{M-1,2} x_{M+1,2}\right) & \text { for } n=M .\end{cases}
$$

Therefore

$$
\sum_{n=1}^{M}\left|B_{n}^{M}-B_{n}\right|^{2}=\left|B_{M-1}^{M}-B_{M-1}\right|^{2}+\left|B_{M}^{M}-B_{M}\right|^{2}
$$

so

$$
\lim _{M \rightarrow \infty} \int \sum_{n=1}^{M}\left|B_{n}^{M}-B_{n}\right|^{2} d \mu^{\beta, v} \leq \lim _{M \rightarrow \infty} \frac{8}{v^{2}}\left[\frac{a^{2}}{\lambda^{2 \beta}} k_{M}^{2-4 \beta}+\frac{a^{2}}{\lambda^{2 \beta}} k_{M+1}^{2-4 \beta}+b^{2} k_{M}^{2-4 \beta}\right]=0 .
$$

This holds for $\beta>\frac{1}{2}$.

Moreover we have (see [4])

Lemma 4.1 For any $\alpha_{1}, \alpha_{2}, \alpha_{3} \in \mathbb{R}$

$$
B: H^{\alpha_{1}} \times H^{\alpha_{2}} \rightarrow H^{-\alpha_{3}} \text { with } \alpha_{1}+\alpha_{2}+\alpha_{3} \geq 1
$$

and there exists a constant $c$ (depending on $a, b, \lambda$ and the $\alpha_{j}$ 's) such that

$$
\|B(u, v)\|_{-\alpha_{3}} \leq c\|u\|_{\alpha_{1}}\|v\|_{\alpha_{2}} \quad \forall u \in H^{\alpha_{1}}, v \in H^{\alpha_{2}} .
$$

This implies that conditions (C5) are true: (6) for any $\frac{\beta}{2} \leq \alpha<\beta$ and (7) for any $\alpha$.

We finally point out that our results of Sect. 3.2 hold also in any space $\mathcal{L}^{p}\left(\mu^{\beta, v}\right)$ with $p=1,2, \ldots$ (see Remark 3.10). Indeed, we have

$$
\int\left|B_{n}(x, x)\right|^{q} \mu^{\beta, v}(d x)<\infty \quad \forall n, q \in \mathbb{N}
$$

for $q=2$ this is (4) of (C3).

Remark 4.2 The stochastic 2D Navier-Stokes equation and the deterministic unforced 2D Euler equation can be represented by Eqs. (13) and (14) respectively ( $-A$ is the Stokes operator and $B(u, v)$ is related to the term $(u \cdot \nabla) v)$. However, not all assumptions $(\mathrm{C} 1)-$ (C5) are fulfilled. Indeed, (C1) holds true, (C2) holds true for $\beta=1$ (the peculiarity of 2D 
hydrodynamics, and the conserved quantity $S_{1}$ is the enstrophy); for $\beta=1$ (the choice is imposed by (C2)) condition (4) of (C3) holds. Condition (C4) holds if modified allowing $\alpha<0$; this makes a big change in the analysis of existence and uniqueness of solutions in the space $H^{\alpha}$ (see $[2,13,16]$ ). Condition (C5) changes, but the new estimate allows anyway to get existence and uniqueness of solution.

Hence, we can conclude that with some modifications a similar set of conditions is fulfilled by the stochastic 2D Navier-Stokes equation and the deterministic unforced 2D Euler equation, except condition (5) of (C3). This reflects the fact that for the 2D Navier-Stokes equation with space-time white noise it has been proven existence and uniqueness of the solution for $\mu^{1, v}$-almost every initial data, and that $\mu^{1, v}$ is an invariant measure, but no results on the uniqueness of the invariant measure are known.

\section{Inviscid Models}

We are interested in the deterministic inviscid and unforced dynamics represented by Eq. (14). We have that the measure $\mu^{\beta, v}$ is infinitesimally invariant for Eq. (14); now $\left(L, F C_{b}^{\infty}\right)$ is the Liouville operator associated to the deterministic inviscid equation (14). Hence, the problem is to see if there exists a global dynamic leaving $\mu^{\beta, v}$ invariant.

Here, we present our results for the SABRA shell model with $\beta=1$ (the physical relevant case) only to make simpler the exposition, but it can be generalized to the other fluid dynamicals models.

Equation (14) is formally obtained from Eq. (13) setting $v=0$ and considering a vanishing right hand side. More generally, we can consider the nonlinear viscous equation

$$
d u^{\varepsilon}(t)+\left[v \varepsilon A u^{\varepsilon}(t)+B\left(u^{\varepsilon}(t), u^{\varepsilon}(t)\right)\right] d t=\sqrt{2 \varepsilon} d w(t), \quad t>0,
$$

with $\varepsilon>0$. When $\varepsilon=0$ we get Eq. (14) (with $\beta=1$ ). Our results of the previous sections hold true for any $\varepsilon>0$.

Therefore, we shall consider the limit $\varepsilon \rightarrow 0$ in the viscous stochastic case to get the inviscid deterministic one (see also a similar case studied in [22]).

The fact that the measure $\mu^{1, v}$ is an invariant measure for any $\varepsilon>0$ can be easily checked. We proceed as in the previous section, but now the Kolmogorov operator associated to Eq. (61) is $K^{\varepsilon}=\varepsilon Q+L$; bearing in mind (37) and (38) we get that $\mu^{1, v}$ is an infinitesimal invariant measure for the operator $\left(K^{\varepsilon}, F C_{b}^{\infty}\right)$. And for any $\varepsilon>0$ the operator $\left(K^{\varepsilon}, F C_{b}^{\infty}\right)$ is dissipative on $\mathcal{L}^{p}\left(\mu^{1, \nu}\right)$ for any $1 \leq p<\infty$.

We are going to prove that when the initial data is a random variable with law $\mu^{1, \nu}$, then Eq. (14) has a solution which is a stationary random process, whose law at any fixed time is $\mu^{1, v}$.

An important property is the integrability of $B$ with respect to the measure $\mu^{1, \nu}$.

Proposition 5.1 If $v>0$, then for any $\alpha<1$ we have

$$
\int\|B(x, x)\|_{\alpha}^{p} \mu^{1, v}(d x)<\infty
$$

for any $p \in \mathbb{N}$. 
Proof We write the proof for $p=2$ but it is the same for the other values of $p$, since $\mu^{1, v}$ is Gaussian and the $B_{n}$ 's are second order polynomial. We have

$$
\begin{aligned}
\int\left|B_{n, 1}(x, x)\right|^{2} \mu^{1, v}(d x)= & \int \mid a k_{n+1}\left[-x_{n+1,2} x_{n+2,1}+x_{n+1,1} x_{n+2,2}\right] \\
& +b k_{n}\left[-x_{n-1,2} x_{n+1,1}+x_{n-1,1} x_{n+1,2}\right] \\
& +\left.(a+b) k_{n-1}\left[x_{n-1,2} x_{n-2,1}+x_{n-1,1} x_{n-2,2}\right]\right|^{2} \mu^{1, v}(d x) \\
\leq & 2 \int\left\{a^{2} k_{n+1}^{2}\left[x_{n+1,2}^{2} x_{n+2,1}^{2}+x_{n+1,1}^{2} x_{n+2,2}^{2}\right]\right. \\
& +b^{2} k_{n}^{2}\left[x_{n-1,2}^{2} x_{n+1,1}^{2}+x_{n-1,1}^{2} x_{n+1,2}^{2}\right] \\
& \left.+(a+b)^{2} k_{n-1}^{2}\left[x_{n-1,2}^{2} x_{n-2,1}^{2}+x_{n-1,1}^{2} x_{n-2,2}^{2}\right]\right\} \mu^{1, v}(d x) \\
= & \frac{16}{v^{2}}\left\{a^{2} k_{n+1}^{2}\left(\lambda_{n+1} \lambda_{n+2}\right)^{-1}+b^{2} k_{n}^{2}\left(\lambda_{n-1} \lambda_{n+1}\right)^{-1}\right. \\
& \left.+(a+b)^{2} k_{n-1}^{2}\left(\lambda_{n-1} \lambda_{n-2}\right)^{-1}\right\} \\
= & \frac{4}{v^{2} k_{0}^{2}}\left\{a^{2} \lambda^{-4}+b^{2}+(a+b)^{2} \lambda^{4}\right\} \lambda^{-2 n} .
\end{aligned}
$$

Similarly, we estimate $\int\left|B_{n, 2}(x, x)\right|^{2} \mu^{1, v}(d x)$. Therefore

$$
\begin{aligned}
\int\|B(x, x)\|_{\alpha}^{2} \mu^{1, v}(d x) & =\int \sum_{n=1}^{\infty} \lambda_{n}^{\alpha}\left|B_{n}(x, x)\right|^{2} \mu^{1, v}(d x) \\
& \leq c_{v, k_{0}, \lambda}\left(|a|^{2}+|b|^{2}\right) \sum_{n=1}^{\infty} \lambda^{2 n(\alpha-1)}
\end{aligned}
$$

which is finite if $\alpha<1$.

Here is our main result.

Theorem 5.2 For any $v>0$, there exists a $\mu^{1, v}$-stationary process, whose paths solve Eq. (14) $\mathbb{P}$-a.s. In particular, the paths are in $C^{\delta}\left(\mathbb{R} ; H^{\alpha}\right)\left(\right.$ for any $0 \leq \delta<\frac{1}{2}$ and $\left.\alpha<1\right)$.

Proof We fix $v>0$ arbitrarily. According to Corollary 3.8, Eq. (61) has a unique $\mu^{1, v_{-}}$ stationary solution $\bar{v}^{\varepsilon}$; this process is a strong solution and has paths in $C\left([0, \infty) ; H^{\alpha}\right)$ a.s. (for $\alpha<1$, but we always think of $\alpha$ as much close to 1 as possible).

First, we prove that the sequence $\left\{\bar{v}^{\varepsilon}\right\}_{0<\varepsilon \leq 1}$ is tight in $C^{\tilde{\delta}}\left([0, T] ; H^{\tilde{\alpha}}\right)$ for any $\tilde{\delta} \in\left(0, \frac{1}{2}\right)$ and $\tilde{\alpha}<\alpha$.

We write Eq. (61) in the mild form:

$$
\bar{v}^{\varepsilon}(t)=\bar{z}^{\varepsilon}(t)-\int_{0}^{t} e^{-v \varepsilon A(t-s)} B\left(\bar{v}^{\varepsilon}(s), \bar{v}^{\varepsilon}(s)\right) d s,
$$

where

$$
\bar{z}^{\varepsilon}(t)=e^{-\nu \varepsilon A t} \bar{v}^{\varepsilon}(0)+\int_{0}^{t} e^{-\nu \varepsilon A(t-s)} \sqrt{2 \varepsilon} d w(s)
$$


is the $\mu^{1, v}$-stationary solution of the linear equation

$$
d z^{\varepsilon}(t)+v \varepsilon A z^{\varepsilon}(t) d t=\sqrt{2 \varepsilon} d w(t)
$$

with the initial data of law $\mu^{1, \nu}$.

We consider the two terms in the right hand side of (62). Using the $\mu^{1, v}$-stationarity we have that for any $0 \leq \delta<\frac{1}{2}$ there exists a constant $\bar{C}_{\delta}>0$ such that

$$
\sup _{0<\varepsilon \leq 1} \mathbb{E}\left[\left\|\bar{z}^{\varepsilon}\right\|_{C^{\delta}\left([0, T] ; H^{\alpha}\right)}\right] \leq \bar{C}_{\delta}
$$

We take $\eta \in(0,1)$ and set $\gamma=\alpha-2 \eta$. For the convolution integral in (62) we have

$$
\begin{aligned}
& \left\|\int_{0}^{\cdot} e^{-\nu \varepsilon A(\cdot-s)} B\left(\bar{v}^{\varepsilon}(s), \bar{v}^{\varepsilon}(s)\right) d s\right\|_{W^{1, p}\left(0, T ; H^{\gamma}\right)}^{p} \\
& =\int_{0}^{T}\left\|\int_{0}^{t} e^{-v \varepsilon A(t-s)} B\left(\bar{v}^{\varepsilon}(s), \bar{v}^{\varepsilon}(s)\right) d s\right\|_{\gamma}^{p} d t+\int_{0}^{T}\left\|B\left(\bar{v}^{\varepsilon}(t), \bar{v}^{\varepsilon}(t)\right)\right\|_{\gamma}^{p} d t \\
& +\int_{0}^{T}\left\|\int_{0}^{t} v \varepsilon A e^{-\nu \varepsilon A(t-s)} B\left(\bar{v}^{\varepsilon}(s), \bar{v}^{\varepsilon}(s)\right) d s\right\|_{\gamma}^{p} d t \\
& \leq \int_{0}^{T} t^{p-1}\left(\int_{0}^{t}\left\|e^{-\nu \varepsilon A(t-s)} B\left(\bar{v}^{\varepsilon}(s), \bar{v}^{\varepsilon}(s)\right)\right\|_{\gamma}^{p} d s\right) d t+\int_{0}^{T}\left\|B\left(\bar{v}^{\varepsilon}(t), \bar{v}^{\varepsilon}(t)\right)\right\|_{\gamma}^{p} d t \\
& +v \varepsilon \int_{0}^{T}\left(\int_{0}^{t}\left\|A e^{-v \varepsilon A(t-s)} B\left(\bar{v}^{\varepsilon}(s), \bar{v}^{\varepsilon}(s)\right)\right\|_{\gamma} d s\right)^{p} d t \\
& \leq \int_{0}^{T} t^{p-1}\left(\int_{0}^{t}\left\|B\left(\bar{v}^{\varepsilon}(s), \bar{v}^{\varepsilon}(s)\right)\right\|_{\gamma}^{p} d s\right) d t+\int_{0}^{T}\left\|B\left(\bar{v}^{\varepsilon}(t), \bar{v}^{\varepsilon}(t)\right)\right\|_{\gamma}^{p} d t \\
& +v \varepsilon \int_{0}^{T}\left(\int_{0}^{t}\left\|A^{1-\eta} e^{-v \varepsilon A(t-s)} A^{\eta} B\left(\bar{v}^{\varepsilon}(s), \bar{v}^{\varepsilon}(s)\right)\right\|_{\gamma} d s\right)^{p} d t \\
& \leq\left(\frac{1}{p} T^{p}+1\right) \int_{0}^{T}\left\|B\left(\bar{v}^{\varepsilon}(t), \bar{v}^{\varepsilon}(t)\right)\right\|_{\gamma}^{p} d t \\
& +v \varepsilon \int_{0}^{T}\left(\int_{0}^{t} c_{p, v} \frac{\left\|B\left(\bar{v}^{\varepsilon}(s), \bar{v}^{\varepsilon}(s)\right)\right\|_{\alpha}}{(t-s)^{1-\eta}} d s\right)^{p} d t \quad \text { by (3). }
\end{aligned}
$$

For the latter integral we use Hölder inequality and get that

$$
\begin{aligned}
& \left(\int_{0}^{t} \frac{\left\|B\left(\bar{v}^{\varepsilon}(s), \bar{v}^{\varepsilon}(s)\right)\right\|_{\alpha}}{(t-s)^{1-\eta}} d s\right)^{p} \\
& \quad \leq\left(\int_{0}^{t} \frac{d s}{(t-s)^{1-\frac{\eta}{2}}}\right)^{2 p \frac{1-\eta}{2-\eta}}\left(\int_{0}^{t}\left\|B\left(\bar{v}^{\varepsilon}(s), \bar{v}^{\varepsilon}(s)\right)\right\|_{\alpha}^{\frac{2}{\eta}-1} d s\right)^{p \frac{\eta}{2-\eta}} .
\end{aligned}
$$

Hence, for $p>\frac{2}{\eta}-1$ we have

$$
\left\|\int_{0}^{\cdot} e^{-v \varepsilon A(\cdot-s)} B\left(\bar{v}^{\varepsilon}(s), \bar{v}^{\varepsilon}(s)\right) d s\right\|_{W^{1, p}\left(0, T ; H^{\gamma}\right)}^{p}
$$




$$
\leq\left(\frac{1}{p} T^{p}+1\right) \int_{0}^{T}\left\|B\left(\bar{v}^{\varepsilon}(t), \bar{v}^{\varepsilon}(t)\right)\right\|_{\gamma}^{p} d t+v \varepsilon T^{m} \int_{0}^{T}\left\|B\left(\bar{v}^{\varepsilon}(t), \bar{v}^{\varepsilon}(t)\right)\right\|_{\alpha}^{p} d t
$$

for some positive constant $m=m_{\eta, v, p}$.

Integrating with respect to the measure $\mu^{\beta, v}$ and using the invariance we get

$$
\begin{aligned}
& \mathbb{E}\left\|\int_{0} e^{-v \varepsilon A(\cdot-s)} B\left(\bar{v}^{\varepsilon}(s), \bar{v}^{\varepsilon}(s)\right) d s\right\|_{W^{1, p}\left(0, T ; H^{\gamma}\right)}^{p} \\
& \quad \leq T\left(1+\frac{1}{p} T^{p}+v \varepsilon T^{m}\right) \int\|B(x, x)\|_{\alpha}^{p} \mu^{1, v}(d x) .
\end{aligned}
$$

Now, we use that $W^{1, p}(0, T) \subset C^{\delta}([0, T])$ if $1-\frac{1}{p}>\delta$. Then, using the previous estimates in (62), given any $0 \leq \delta<\frac{1}{2}, p>\frac{1}{1-\delta}$ and $p>\frac{2}{\eta}-1$ we have

$$
\sup _{0<\varepsilon \leq 1} \mathbb{E}\left[\left\|\bar{v}^{\varepsilon}\right\|_{C^{\delta}\left([0, T] ; H^{\gamma}\right)}^{p}\right]<\infty
$$

On the other hand, the space $C^{\delta}\left([0, T] ; H^{\gamma}\right)$ is compactly embedded in $C^{\tilde{\delta}}\left([0, T] ; H^{\tilde{\gamma}}\right)$ if $\tilde{\delta}<\delta$ and $\tilde{\gamma}<\gamma$; this follows from the compact embedding $H^{\gamma} \Subset H^{\tilde{\gamma}}$ and from the AscoliArzelà theorem. Because these results hold for any $\delta \in\left[0, \frac{1}{2}\right.$ ) and $\tilde{\gamma}<\gamma<\alpha<1$ (with $p$ big enough, but we use (60)), we can consider any $\tilde{\delta}<\frac{1}{2}$ and any $\tilde{\gamma}<1$. The tightness follows from (67) as usual by means of Chebyshev inequality. And to simplify notation henceforth we consider the tightness in the space $C^{\delta}\left([0, T] ; H^{\alpha}\right)\left(\delta<\frac{1}{2}\right.$ and $\left.\alpha<1\right)$.

By the tightness result and Prohorov theorem, the sequence of the laws of $\bar{v}^{\varepsilon}$ has a subsequence $\left\{\bar{v}^{\varepsilon_{n}}\right\}_{n=1}^{\infty}$ weakly convergent as $n \rightarrow \infty$ (with $\varepsilon_{n} \rightarrow 0$ ) in $C^{\delta}\left([0, T] ; H^{\alpha}\right)$ to some limit measure. By a diagonal argument, this holds for any $T$ and therefore the limit measure leaves in $C^{\delta}\left([0, \infty) ; H^{\alpha}\right)$. By Skorohod theorem, there exist a probability space $(\tilde{\Omega}, \tilde{\mathbb{F}}, \tilde{\mathbb{P}})$, a random variable $\tilde{v}$ and a sequence $\left\{\tilde{v}^{\varepsilon}\right\}$ such that $\operatorname{law}\left(\tilde{v}^{\varepsilon}\right)=\operatorname{law}\left(\bar{v}^{\varepsilon}\right)$, $\operatorname{law}(\tilde{v})=\mu^{1, v}$ and $\tilde{v}^{\varepsilon}$ converges to $\tilde{v}$ a.s. in $C^{\delta}\left([0, \infty) ; H^{\alpha}\right)$.

We now identify the equation satisfied by $\tilde{v}$. We are going to prove that $\tilde{\mathbb{P}}$-almost each path solves (14).

It is enough to control the behavior of the terms with $B$. First

$$
\begin{aligned}
& e^{-\nu \varepsilon A(t-s)} B\left(\tilde{v}^{v, \varepsilon}(s), \tilde{v}^{v, \varepsilon}(s)\right)-B\left(\tilde{v}^{v}(s), \tilde{v}^{v}(s)\right) \\
& =e^{-v \varepsilon A(t-s)}\left[B\left(\tilde{v}^{v, \varepsilon}(s), \tilde{v}^{v, \varepsilon}(s)\right)-B\left(\tilde{v}^{v}(s), \tilde{v}^{v}(s)\right)\right] \\
& \quad+\left[e^{-\nu \varepsilon A(t-s)}-I\right] B\left(\tilde{v}^{v}(s), \tilde{v}^{v}(s)\right) .
\end{aligned}
$$

When we consider the second addend in the mild form expression, it trivially converges to zero; but for the convergence of the first one it is enough to verify that

$$
\int_{0}^{t}\left\|B\left(\tilde{v}^{v, \varepsilon}(s), \tilde{v}^{v, \varepsilon}(s)\right)-B\left(\tilde{v}^{v}(s), \tilde{v}^{v}(s)\right)\right\|_{\alpha-1} d s \rightarrow 0
$$

as $\varepsilon \rightarrow 0$; for this we use the bilinearity and the estimate (11).

Similarly we work on the time interval $[-T, 0]$ by considering the reversed-time parabolic nonlinear equation

$$
d u^{\varepsilon}(t)+\left[-v \varepsilon A u^{\varepsilon}(t)+B\left(u^{\varepsilon}(t), u^{\varepsilon}(t)\right)\right] d t=\sqrt{2 \varepsilon} d w(t), \quad t<0 .
$$


It has a unique $\mu^{1, v}$-stationary solution $\underline{v}^{\varepsilon}$; this process is a strong solution, has paths in $C^{\delta}\left((-\infty, 0] ; H^{\alpha}\right)$. The tightness and the convergence are obtained in the same way as above.

Acknowledgements We are very grateful to an anonymous referee that helped in making better the exposition of this paper. The work of H. Bessaih was partially supported by the NSF grant No. DMS 0608494, and by GNAMPA-INDAM project. We would like to also acknowledge the hospitality of EPFL Lausanne where some parts of this paper have been refined while the authors were visiting the Bernoulli Center.

\section{References}

1. Albeverio, S., Cruzeiro, A.B.: Global flows with invariant (Gibbs) measures for Euler and Navier-Stokes two-dimensional fluids. Commun. Math. Phys. 129(3), 431-444 (1990)

2. Albeverio, S., Ferrario, B.: Uniqueness of solutions of the stochastic Navier-Stokes equation with invariant measure given by the enstrophy. Ann. Probab. 32(2), 1632-1649 (2004)

3. Barbato, D., Barsanti, M., Bessaih, H., Flandoli, F.: Some rigorous results on a stochastic GOY model. J. Stat. Phys. 125(3), 677-716 (2006)

4. Bessaih, H., Ferrario, B.: Invariant Gibbs measures of the energy for shell models of turbulence; the inviscid and viscous cases. Nonlinearity 25, 1075-1097 (2012)

5. Bessaih, H., Millet, A.: Large deviation principle and inviscid shell models. Electron. J. Probab. 14(89), 2551-2579 (2009)

6. Boffetta, G., Ecke, R.E.: Two-dimensional turbulence. Annu. Rev. Fluid Mech. 44, 427-451 (2012)

7. Bouchet, F., Corvellec, M.: Invariant measures for the 2D Euler and Vlasov equations. J. Stat. Mech. 08, P08021 (2010)

8. Chojnowska-Michalik, A., Goldys, B.: Symmetric Ornstein-Uhlenbeck semigroups and their generators. Probab. Theory Relat. Fields 124(4), 459-486 (2002)

9. Chueshov, I., Millet, A.: Stochastic 2D hydrodynamical type systems: well posedness and large deviations. Appl. Math. Optim. 61(3), 379-420 (2010)

10. Constantin, P., Levant, B., Titi, E.S.: Analytic study of shell models of turbulence. Physica D 219(2), 120-141 (2006)

11. Da Prato, G.: Kolmogorov Equations for Stochastic PDEs. Advances Courses in Mathematics CRM Barcelona. Birkhäuser, Basel (2004)

12. Da Prato, G.: An Introduction to Infinite-Dimensional Analysis. Springer, Berlin (2006)

13. Da Prato, G., Debussche, A.: Two-dimensional Navier-Stokes equations driven by a space-time white noise. J. Funct. Anal. 196(1), 180-210 (2002)

14. Da Prato, G., Zabczyk, J.: Stochastic Equations in Infinite Dimensions. Encyclopedia of Mathematics and Its Applications, vol. 44. Cambridge University Press, Cambridge (1992)

15. Da Prato, G., Zabczyk, J.: Ergodicity for Infinite Dimensional Systems. LMS Lecture Notes, vol. 229. Cambridge University Press, Cambridge (1996)

16. Debussche, A.: The 2D-Navier-Stokes equations perturbed by a delta correlated noise. In: Probabilistic Methods in Fluids, pp. 115-129. World Scientific, River Edge (2003)

17. Eberle, A.: Uniqueness and Non-uniqueness of Semigroups Generated by Singular Diffusion Operators. Lecture Notes in Mathematics, vol. 1718. Springer, Berlin (1999)

18. Ferrario, B.: A note on a result of Liptser-Shiryaev. Stoc. Anal. Appl. (2012). doi:10.1080/07362994. 2012.727139. Eprint arXiv: $1005.0237 \mathrm{v} 2$

19. Ferrario, B.: Uniqueness and absolute continuity for semilinear SPDE's. In: The Proceedings of Ascona 2011. Progress in Probability. Birkhäuser, Basel (2012, to appear)

20. Flandoli, F.: An introduction to 3D stochastic fluid dynamics. In: Da Prato, G., Röckner, M. (eds.) SPDE in Hydrodynamic: Recent Progress and Prospects, pp. 51-150. Lectures Given at the C.I.M.E. Summer School Held in Cetraro, August 29-September 3, 2005. Lecture Notes in Math., vol. 1942. Springer, Berlin; Fondazione C.I.M.E., Florence (2008)

21. Gledzer, E.B.: System of hydrodynamic type admitting two quadratic integrals of motion. Dokl. Akad. Nauk SSSR 209, 1046-1048 (1973). Engl. transl.: Sov. Phys. Dokl. 18, 216-217 (1973)

22. Kuksin, S.B.: The Eulerian limit for 2 D statistical hydrodynamics. J. Stat. Phys. 115(1-2), 469-492 (2004)

23. Kuo, H.H.: Gaussian Measures in Banach Spaces. Lecture Notes in Mathematics, vol. 463. Springer, Berlin (1975) 
24. L’vov, V.S., Podivilov, E., Pomyalov, A., Procaccia, I., Vandembroucq, D.: Improved shell model of turbulence. Phys. Rev. E 58, 1811-1822 (1998)

25. Ohkitani, K., Yamada, M.: Lyapunov spectrum of a chaotic model of three-dimensional turbulence. J. Phys. Soc. Jpn. 56, 4210-4213 (1987)

26. Pazy, A.: Semigroups of Linear Operators and Applications to Partial Differential Equations. Applied Mathematical Sciences, vol. 44. Springer, New York (1983)

27. Temam, R.: Navier-Stokes Equations. Theory and Numerical Analysis. Studies in Mathematics and Its Applications, vol. 2. North-Holland, Amsterdam (1984) 